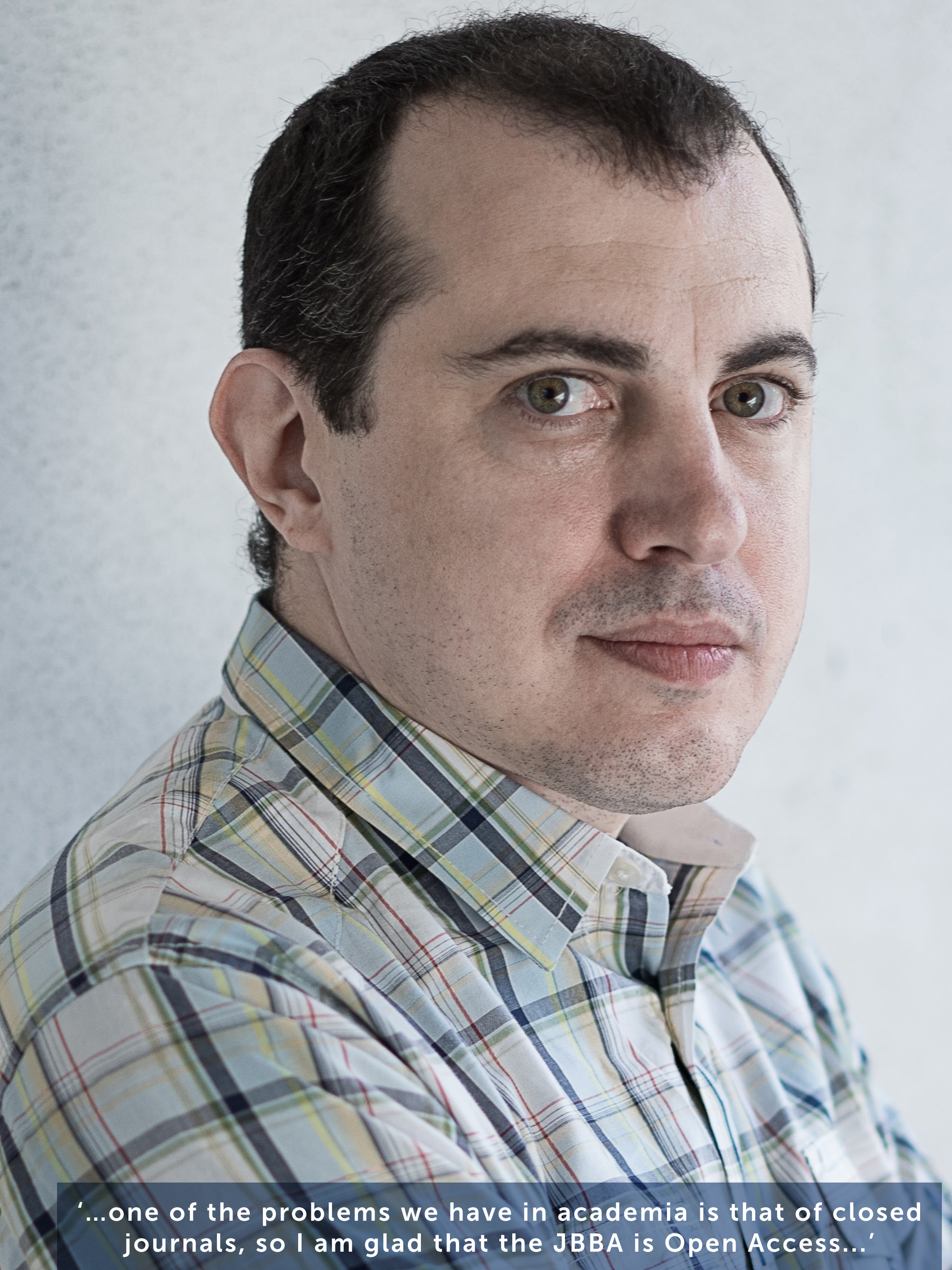




\section{INTERVIEW WITH}

\section{ANDREAS M. ANTONOPOULOS}

Author of Mastering Bitcoin, The Internet of Money Series and Mastering Ethereum

You have spoken to hundreds and thousands of people around the globe about Bitcoin and the Blockchain Technology. What in your opinion, has over the years been some of the biggest challenges as an educator of this technology?

The first and the biggest challenge is the lack of financial education worldwide which makes it necessary for me to start the discussion with what is money, before I can get to what Bitcoin is. For most people money is a very abstract concept and one that is very poorly understood. Most people have a very little understanding of how the modern monetary system works, what its components are and how money works in reality. Instead, what they have is a mythology, a substituting fact, which in many cases is a very naive understanding - and that's detrimental to understanding the economy, the political power of money and the influence of certain organizations. So explaining money is hard.

Secondly, while trying to explain that Bitcoin was broader than simply a payments network, across the industry we all adopted and started using the term Blockchain to express the concept of more than just a payment network and that term was then hijacked by the marketing professionals trying to promote various companies that have very little to do with what the term Blockchain actually means, diluted to the point of it being meaningless. The efforts to generalize and have a broader term that applies to the broader technology beyond just Bitcoin, has given us a very diluted term that means nothing and kind of martialing that pact and giving people the tools to discern what is really relevant to the term Blockchain and what is not.

So for example, you hear people say things like Blockchains are immutable, which are not. Some Blockchains are immutable not all. And to kind of reflect onto the term Blockchains, all of these benefits or features which are really not benefits and features of Blockchain in general, but only certain Blockchains, in certain ways with certain characteristics, architecture and design choices.

Also, this is a technology that threatens the status quo and it also represents a very big fundamental change in what money is and how it's used and who gets to use it and that means that both the term Bitcoin and the term Blockchain are also wrapped in misunderstanding and deliberate misinformation, in order to discredit the technology for political reasons.

You mentioned about a lot of misunderstandings and hype so how could policy makers and decision makers separate facts from fiction and what lessons can we learn from the journey to date in terms of separating this hype from reality?

One of the most popular talks I've done is called 'Blockechain vs. Bullshit' which discusses exactly that topic. Blockchain describes the data structure and really the most important invention that Bitcoin brought to the world wasn't the Blockchain data structure itself, but rather it was the consensus algorithm, proof of work, and combination of the technologies that allow decentralisation to emerge.

Decentralisation is a powerful property; the ability to run a system where no one is directly in charge or in control, to take power and spread it across multiple participants so that they don't have to trust each other or trust any central authority; i.e. you decentralize the power.

Neutrality, meaning that it doesn't matter who the sender, recipient, or the value is; it treats everyone similar. The borderless nature of the system depends on decentralisation, otherwise it would be subjected to hundreds of regulatory domains and not be truly borderless.

The censorship resistance, which means no one can stop a transaction from happening or reverse it once it has happened. The open access nature of public blockchains mean that anyone can participate, without identity, without proof, without verification, in a truly open, borderless, neutral and censorship resistant way.

What role, in your opinion, scholars and researches can play in advancing this technology and establishing a body of scientific evidence and also disseminating authentic information?

Well, I think what we've seen, which is rather interesting, 
is an explosion in the amount of the academic research that's happening, not just in the area of the Blockchain, but other consensus algorithms that didn't exist before. But more broadly, I think the invention of Bitcoin has ushered in a new golden era of Cryptography. Applied cryptography, which on a global scale endeavors to do things that cypherpunks have been imagining and envisioning for fifty years, are now being used by average people. Cryptography has never been more practical and more applied than since the invention of Bitcoin. Bitcoin and its cousins, all of the other open blockchains, are now the largest deployments of public key cryptography on the planet and in history. We are probably soon going to surpass the planets largest deployment of PKC which is the military, so civilians will have practical use of cryptographic tools for the first time in excess of a government's military.

Bitcoin has pushed the state of the art in public key cryptography, elliptic curve, digital signatures and trusted systems research and so it's feeding academia and benefitting back from the results.

For MSc and $\mathrm{PhD}$ students who are keen to explore Blockchain research (dissertation and thesis) and are looking for ideas, what advice would you give and what are the areas that need further academic investigations to explore the technology?

The greatest areas of opportunity for research lies in the fact that we have a live production level, deployed, systems and infrastructure for monetary cryptography. This is producing real time data every single day and is in a giant experimental sandbox with real money at stake which has never happened before. So, there is a great opportunity to use that as a research tool.

How would you like to see the role of scholarly journal like the JBBA supporting Blockchain and Cryptocurrency research to build some frameworks and benchmarks and good scientific body of evidence?

I think it's important to recognize that this invention came out of the open source culture of the internet, the creative commons culture, and that one of the problems we have in academia is that of closed journals. I think, it is unethical to work in the open Blockchain space (to build on shoulders of the giants as they say), to build on top of technologies developed in the open source community and derived from the open source culture and then do closed proprietary research. I think that is unethical, to take what others have given freely and not give back, so I am glad that the JBBA is Open Access.

To establish Bitcoin and Blockchain as a subject in terms of scientific discipline, we have seen a huge interest from students and scholars from different backgrounds; Computer Sciences, Economics, Information Technology etc., what steps in your opinion should be taken by policy makers and universities for this to become fully integrated in the curriculum?

Blockchain is still a new scientific domain and is still in the very early stages of maturity. I think, until it matures as a technology and as a scientific discipline, it is more appropriate to deal with it at the postgraduate and not the undergraduate level. It represents the front end of a multidisciplinary research topic and therefore it should be approached by academia as an opportunity to take the existing knowledge and extend it, establish better foundations and flush out the details, instead of trying to teach it to undergraduates as an established science since the things are moving too fast. It is heavily research based, so it is more appropriate for the $\mathrm{PhD}$ level exploration and somewhat appropriate for the multidisciplinary MSc's.

How important it is for general public to have an understanding of blockchain and how in-depth should it be?

Users and public should learn a bit of new vocabulary and then the rest should be intuitive; we need to make it intuitive enough. The requirement today to have an in-depth understanding of the technology before one could really apply it, is a demonstration of our failure to deliver mature user interfaces, design paradigms and consumer experiences. Our failure to make the technology intuitive enough is simply a reflection of the early stage lack of maturity. And that's okay; that's a natural evolution of any new technology.

The BBA is now one of the biggest and the most prestigious Blockchain Associations in the world. We have got advisors and members in six continents and an extended student network. Any advice on how we can support the next generations of Blockchain professionals?

I think some interesting things have happened in terms of emergence of various organisations and associations. Because of the nature of this technology and the importance of decentralisation, I think that the 
most important decisions are still local decisions and the most important perspective is still local perspective. You cannot as a British academic tell a Nigerian farmer how to use this technology. You cannot even understand their prospective, neither I can. I have been doing this now for 6 years fulltime; I travel all around the world and as I travel and talk about this topic and meet communities, I learned that this technology means different things to different communities based on their needs, their environment, their culture, their language, their economic circumstances, their former government, their perception of money, their perception of government and their practical needs.

Bitcoin is very different to an Argentinian than it is to a New Yorker. It is very different to a Venezuelans than it is to a Londoner and it is very different to Chinese than it is to a Westerner. That applies to open Blockchain technologies as well.

\section{And finally, when is Mastering Ethereum coming} out?

Tentatively December $1^{\text {st }}$, probably before December $15^{\text {th }}$ I'm getting the copy edited version back from the publisher this weekend then it's going into final production. It's going to take about 5 weeks to produce and then it should start printing. It's definitely going to happen in 2018 !

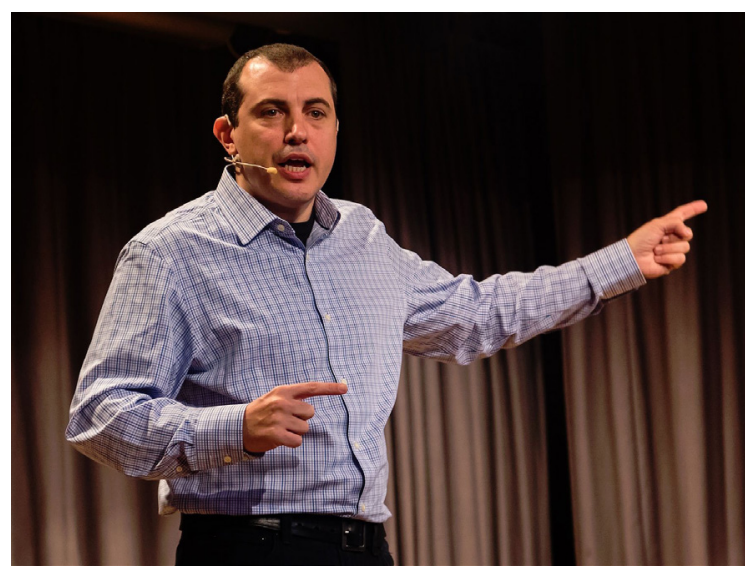

Andreas M. Antonopoulos at one of the conferences. All his work is available for free and under open creative commons licenses and is supported by his supporters and patrons. 